\section{Kirchhoff, Gustav Robert}

T. Arndt

Bioscientia Institut für Medizinische Diagnostik $\mathrm{GmbH}$, Ingelheim, Deutschland

Lebensdaten Deutscher Physiker, geboren am 12. März 1824 in Königsberg (Preußen), gestorben am 17. Oktober 1887 in Berlin

Verdienste Entwicklung der Spektralanalyse gemeinsam mit Bunsen, die er auch auf die Sonnenstrahlung ausdehnte.
Beschreibt die Gesetze der Lichtabsorption und -emission sowie der Stromflüsse in verzweigten elektrischen Leitern. Formulierung der Kirchhoffschen Regeln (1. und 2. Kirchhoffsches Gesetz). Studium der Mathematik und Physik in Königsberg. 1848 Dozent in Berlin, 1850 Professor für Experimentalphysik in Breslau. Ab 1854 in Heidelberg.

\section{Literatur}

https://www.kip.uni-heidelberg.de/oeffwiss/kirchhoff; Zugegriffen am 15.09.2017 